\title{
O EMPREGO DE MÉTODOS E TECNOLOGIAS NA BUSCA POR ENGAJAMENTO EM MOOCS
}

SAO PAULO/SP JULHO/2018

\author{
Alessandra Fabiana Cavalcante - UNICID - alessandra.cavalcante@unicid.edu.br \\ Alberto Messias Costa - UNICSUL - alberto.souza@cruzeirodosul.edu.br \\ Luis Naito Mendes Bezerra - UNICSUL - luis.naito@cruzeirodosul.edu.br \\ Márcia Terra da Silva - UNIP - marcia.terra@uol.com.br \\ Regina Tavares de Menezes - UNICsUL - Regina.menezes@cruzeirodosul.edu.br \\ Vilma Silva Lima - UNICSUL - vilma.lima@cruzeirodosul.edu.br
}

Tipo: Investigação Científica (IC)

Natureza: Relatório Final de Pesquisa

Categoria: Métodos e Tecnologias

Setor Educacional: EDUCAÇÃO SUPERIOR, EDUCAÇÃO CONTINUADA EM GERAL

\section{RESUMO}

O presente artigo pretende expor a investigação científica resultante da manipulação e análise de dados primários reveladores sobre os aspectos quantitativos e qualitativos do curso livre denominado Carreira $S / A$; considerado o terceiro curso massivo de maior expressividade, no que tange ao número de matriculados, já registrado pela literatura deste campo científico. Na oportunidade, empenhou-se aferição e comparação dos diferentes níveis de participação dos estudantes na plataforma de gestão e hospedagem de vídeos Kaltura, integrada ao LMS Blackboard.

Palavras-chave: Massive Online Open Courses (MOOCs); Quiz; Curso livre; Videoaulas 


\section{Introdução}

No âmbito da educação superior no Brasil, as matrículas no ensino a distância (EaD) continuam a apresentar crescimento. De acordo com dados recentes do censo realizado pelo Instituto Nacional de Estudos e Pesquisas Educacionais Anísio Teixeira - INEP (2016), quase 1,5 milhão de alunos estão matriculados nesta modalidade no Brasil, com crescimento de 1,2\% em relação ao ano anterior. Vale ressaltar que a EaD apresentou em 2016 uma participação de 18,6\% do total de matrículas da Educação Superior Brasileira. Nos Estados Unidos (EUA), a situação não é diferente. Os últimos dados apontam que no período de 2015 a 2016, a taxa de crescimento do número de estudantes matriculados em pelo menos um curso a distância foi de 5,6\%. Tal segmento apresentou um total de 6.359.121 milhões de alunos matriculados em 2016, que representa $31,6 \%$ dos alunos matriculados no ensino superior americano. (BAKER, ISOTANI, \& DE CARVALHO, 2011; ALLEN \& SEAMAN, 2018).

Uma significativa revolução na modalidade que merece destaque são os Massive Online Open Courses (MOOCs); que estão tendo grande impacto, com potencial para alterar o status quo da educação em todos os sentidos, tanto no nível dos alunos, quanto dos professores e administradores educacionais. No Brasil, as provedoras de mais destaques são: Veduca, da USP, e a eduK. Essas provedoras são as principais, responsáveis, pelas ofertas de MOOcs no território nacional. O modelo brasileiro consiste em cursos pequenos, rápidos, prontos para resolver um dilema, um problema ou desenvolver uma habilidade. Além dos provedores nacionais o Coursera, o edX e a Udacity também ofertam cursos na língua portuguesa.

A partir do surgimento dos MOOCs, ocorreu uma mudança de dimensão a respeito da quantidade de alunos inscritos em um único curso, pois em razão de serem totalmente online sem pré-requisitos e, também, por não exigirem pagamento inicial de taxas, tais cursos têm atraído, em geral, expressivo volume de alunos (HYMAN, 2012; COOPER \& SAHAMI, 2013). O aspecto massivo presente em seu acrônimo pode ser destacado, por exemplo, pelo curso, Introduction to Computer Science I, oferecido pela Universidade de Harvard em parceria com a provedora edX. Esse MOOC teve cerca de 150 mil matrículas. No entanto, essa situação não é comum, ou seja, cursos com mais de 100.000 alunos. Geralmente, cursos dessa natureza apresentam em média 25.000 alunos matriculados. (JORDAN, 2015)

Nos MOOCs, os Ambientes Virtuais de Aprendizagem (AVA) ou LMS (Learning Management System) comerciais e de código aberto, assim como os ambientes virtuais utilizados pelas grandes provedoras como Cousera e edX são o elemento central de 
qualquer projeto. Esses cursos são ministrados de forma "automática", pois são baseados, principalmente, em videoaulas, atividades com correção automática e projetos com avaliação pelos pares, sem um contato direito entre aluno e tutor. Os fóruns de discussão são importantes para apoiar a colaboração entre os pares, permitindo aos alunos obterem informações e, também, interação social com os outros alunos. Apesar de existir uma trilha de aprendizagem previamente definida, os próprios alunos podem gerenciar sua aprendizagem. (NANFITO, 2014; YOU, 2016). Uma análise das ferramentas utilizadas no âmbito dos cursos em formato EaD pode vir a direcionar melhor a relação do aluno com o curso; além de predizer suas dificuldades e oferecer também reforço quando for necessário, sendo portanto um material rico para permitir o autogerenciamento do curso. Diante disso, o referido artigo busca responder a seguinte problemática: qual a efetividade do uso de recursos educacionais, como videoaula e fóruns de discussões, nos cursos a distância. Busca-se, ainda, saber como se dá a relação dos estudantes com essas ferramentas. (PARDO \& KLOOS, 2011; HU, LO, \& SHIH, 2014; NANFITO, 2014; YOU, 2016).

O objetivo do presente estudo é analisar dois aspectos fundamentais relacionados aos recursos educacionais presentes nos projetos da maioria dos cursos a distância, ou seja, videoaulas e fóruns de discussões. Tais análises partirão de estudos realizados a partir dos resultados obtidos de um curso denominado Carreira S/A que teve em sua oferta 181.677 matriculas. Esse curso apresenta uma temática que procurou atender aos interesses de alunos de diversas áreas e cursos de um grupo educacional privado.

Em relação à metodologia utilizada na elaboração deste artigo foi realizada uma revisão bibliográfica a respeito dos principais conceitos pertinentes à $\mathrm{EaD}$ e aos $\mathrm{MOOCs}$, o que resultou na necessidade de desenvolver um curso específico em formato de MOOC e com particularidades que validasse as hipóteses levantadas ao longo da pesquisa.

É importante mencionar que o número de alunos matriculados neste curso, denominado Carreira S/A, o posiciona em lugar de destaque se comparado com MOOCs que tiveram grande quantidade de matrículas. Ao analisar os dados de Jordan (2015), podemos classificá-lo em terceiro lugar, sendo os dois primeiros o Think Again: How to Reason and Argue da provedora Coursera (Duke University), com 226.552 alunos matriculados, e o Social Psychology também da Coursera (Wesleyan University) com 200.000 alunos matriculados.

O curso em questão ainda que não possa ser considerado livre já que não foi liberado para acesso ao público em geral foi considerando como massivo em função do expressivo número grande de alunos matriculados no grupo educacional cuja oferta foi 
disponibilizada. A opção pela criação do curso foi necessária, pois os dados originados nos ambientes virtuais de aprendizagem utilizados pelos MOOCs das principais provedoras como Coursera e Udacity, são de difícil acesso para consulta pública, sendo liberados, apenas para as próprias provedoras e para as instituições de ensino que oferecem os cursos. A iniciativa própria facilita a confirmação dos resultados, pois o ambiente é mais controlado. A oferta de um curso dessa magnitude representa um considerável desafio em termos de gestão, pois essa enorme quantidade de alunos gerou, consequentemente, grande quantidade de dados, além de toda a parte operacional, como verificar os fóruns de discussão e responder as mensagens dos alunos sobre diversos assuntos.

\section{Fundamentação Teórica}

As pesquisas utilizadas como fonte dizem respeitos aos assuntos que emergem do âmbito da educação a distância. Entre eles destacamos os MOOCs. Para desenhar o conceito do curso Carreira S/A e denominá-lo como um MOOC buscamos as orientações presentes na pesquisa de Pomerol, (EPELBOIN \& THORY, 2015). Para eles os principais aspectos de suporte são:

- Necessidade de equipe técnica especializada para a pré-produção, produção e pós-produção de conteúdo na forma de vídeo, assim como postagem e gestão de tal objeto de aprendizagem em plataforma especializada;

- Desenvolvimento de material textual. No contexto dos MOOCs, o conteúdo precisa ser providenciado em sua totalidade por professores conteudistas ou empregados conteúdos que estejam disponíveis na Web no formato Open Educational Resources (OER). É importante ressaltar que a montagem do curso vai além da simples compilação de uma lista de conteúdos;

- Também é necessário providenciar exercícios online no formato de quizzes. Neste sentido, é importante a preocupação em relação ao conteúdo e o formato dos exercícios online, que não têm a mesma flexibilidade dos exercícios utilizados no modelo tradicional; estes corrigidos por professores presencialmente.

Além dos pesquisadores citados acima abrimos destaque, também, para Baturay (2015). Segundo ele, os principais recursos educacionais utilizados no projeto de um MOOC são:

- Videoaulas - normalmente com duração de 5 a 10 minutos, constituem-se como principal recurso utilizado nos MOOCs;

- Avaliação - questões de múltipla-escolha com retorno automático e avaliação 
pelos pares;

- Fórum de discussão - principal mecanismo de interação entre estudantes e professores;

- Leituras - em muitos MOOCs os estudantes não necessitam comprar livros, sendo a maioria dos materiais disponibilizada pelos próprios professores. Contudo, provedores - como a Coursera - podem receber recursos financeiros por meio de programas e convênios com empresas como Amazon;

- Sessões de videoconferência - de forma adicional aos recursos educacionais utilizados em determinado período, é possível incluir sessões de videoconferência com o professor do curso;

- Outros recursos adicionais podem ser oferecidos para o aprofundamento de determinados aspectos do curso.

No que tange aos aspectos relacionados às análises das videoaulas utilizamos os estudos de Guo, Kim \& Rubin (2014). Para estes autores, a qualidade das videoaulas está relacionada ao seu tempo de duração; entre 10 a 15 minutos. Isto pode parecer um curto espaço de tempo, porém, tem sido mostrado que o grau de atenção dado ao assistir a um vídeo diminui rapidamente. Os autores afirmam que o tempo de atenção humana diminui consideravelmente para além de 9 minutos. Assim, as sequências de curso precisam ser divididas, de modo a estar em conformidade com o formato dos vídeos que os alunos estão acostumados a assistir no YouTube.

\section{O curso Carreira S/A}

O curso foi criado e ofertado no âmbito de um grande Grupo Educacional Privado com atuação no Estado de São Paulo e nas regiões Sul e Centro-Oeste do Brasil. A oferta desse curso representou a primeira experiência do grupo com um número de matrículas tão elevado. Além disso, constituiu-se como um projeto piloto para o lançamento de outros cursos no estilo dos MOOCs. Tal curso preservou as principais características dos MOOCs, como não ter cobrança adicional de taxa, ser online, ter o caráter massivo, usar material didático, predominantemente, na forma de vídeo e concentra-se na utilização do fórum de discussão.Neste estudo serão utilizados apenas dados acadêmicos oriundos do Blackboard e do sistema de análise de videoaulas da Plataforma de armazenamento e gestão de vídeos, denominada Kaltura.

\subsection{Características do curso}

O curso Carreira S/A teve duração de 4 semanas (20 de março a 20 de abril de 2017) e oferta no LMS Blackboard, de forma gratuita e automática para todos os alunos 
matriculados nas Instituições do referido Grupo Educacional tanto na modalidade presencial quando EaD. O curso foi composto por 4 unidades de aprendizagem, são elas: Planejamento de Carreira, Competências Profissionais, Processo Seletivo e Recriutamento e Empreendedorismo. O processo de avaliação foi composto por testes de múltipla-escolha e cada unidade correspondeu a $25 \%$ da nota final, ou seja, até 2,5 (dois pontos e meio). Para ser aprovado, o aluno deveria obter nota final igual ou maior que 6,0 (seis). Cada unidade de estudo foi composta pelos seguintes recursos educacionais: videoaula, texto (no formato PDF e livro eletrônico) e fórum de discussão. Para o nosso estudo, no entanto, não efetivaremos análises nos materiais do tipo texto.

\subsection{Análise do material do curso}

4.2.1 Videoaula - o curso utilizou a Kaltura, plataforma totalmente integrada ao LMS Blackboard, disponibilizando ainda uma solução que adapta a reprodução do vídeo ao tipo de dispositivo do usuário (computador desktop, celular e tablet), sendo compatível também com diversos navegadores (browsers) disponíveis no mercado. A Kaltura oferece, também, para os administradores do sistema uma ferramenta para análise dos vídeos (analytics), que foi utilizada para o presente estudo. Na fase de projeto do curso foi considerado que as videoaulas deveriam ter duração média de 7 minutos, sendo estes constituídos por entrevistas e depoimentos de profissionais especializados, além de trechos de filmes, enquetes, animações etc para ilustrar os assuntos tratados. Contudo, por ter sido a primeira versão do curso e devido à quantidade e qualidade do material coletado, a versão final das videoaulas ultrapassou o tempo projetado. A tabela 1 apresenta os dados oriundos da ferramenta Analytics da Kaltura.

\section{Tabela 1: Dados relativos aos vídeos. Fonte: Kaltura Analytics}

\begin{tabular}{|c|c|c|c|c|c|}
\hline Unid & Nome & Duração & Plays & Tempo médio & $\begin{array}{l}\text { Média } \\
\text { (drop-off) }\end{array}$ \\
\hline | & $\begin{array}{l}\text { Planejamento } \\
\text { Carreira }\end{array}$ & de $13: 51$ & 30.159 & $07: 35$ & $54,31 \%$ \\
\hline II & $\begin{array}{l}\text { Competências } \\
\text { Profissionais }\end{array}$ & 13:58 & 20.317 & p8:46 & $62,82 \%$ \\
\hline III & $\begin{array}{l}\text { Processo seletivo } \\
\text { recrutamento }\end{array}$ & e 11:34 & 14.357 & 08:06 & $70,15 \%$ \\
\hline IV & Empreendedorismo & 22:19 & 15.538 & 13:24 & $60,12 \%$ \\
\hline \multicolumn{2}{|c|}{ Total / Média } & & 80.371 & 09:06 & $60,60 \%$ \\
\hline
\end{tabular}


Em relação ao tempo de duração dos vídeos é possível encontrar, na literatura, pesquisas que apontam a média de tempo para reter melhor a atenção dos estudantes. $\mathrm{Na}$ visão de Khan (2012), o tempo ideal para melhorar o engajamento dos estudantes fica entre 10 e 15 minutos. A pesquisa de Khalil \& Ebner (2017) foi direcionada para um MOOC denominado "Social Aspects of Information Technology" ofertado pela provedora iMooX na Áustria, que contou com 21 vídeos de duração média de 17 minutos. Os dados de pesquisa da empresa Kaltura (2016) com 1.500 respondentes (educadores, profissionais especializados em vídeo e alunos) apontaram o intervalo entre 5 e 10 minutos como o mais indicado para a duração de um vídeo. Contudo, é possível encontrar valor inferior como ideal para a duração de um vídeo. Por exemplo, o trabalho de Guo, Kim, \& Rubin (2014) analisou os dados de quatro MOOCs da provedora edXe chegou a conclusão que vídeos de até 6 minutos são muito mais envolventes e retém a atenção dos alunos mais efetivamente.

No caso do curso Carreira S/A, considerando todos as videoaulas, os alunos assistiram, em média, 9min e 6seg, valor que está coerente com a pesquisa da Kaltura (2016) e acima do valor indicado por Guo, Kim, \& Rubin (2014). Os dados ainda apontam que os alunos assistem à aproximadamente $60 \%$ das videoaulas. A partir da experiência dessa primeira edição do curso será possível rever a duração total dos vídeos para a próxima edição.

4.2.2 Fórum de discussão - ainda que não houve um tutor para mediar a participação dos alunos, visto ser essa uma característica comum aos MOOCs, para cada unidade, foi lançado um tema em que os alunos puderam se manifestar e debater a respeito do assunto, usando um modelo de discussão entre os pares para a construção coletiva do conhecimento. Por exemplo, para o primeiro fórum foi sugerida a seguinte questão: você acredita que a elaboração de um plano de carreira pode influenciar a sua trajetória profissional? Ao realizar as análises oriundas dos fóruns percebeu-se uma particularidade entre as modalidades presencial e EaD. No caso dos alunos da modalidade presencial, foram postadas 678 publicações, das quais muitas eram comentários a respeito da questão colocada. No entanto, o espaço foi utilizado, também, para esclarecer dúvidas específicas relacionadas a questões operacionais, como a data de emissão do certificado e, ainda, dúvidas gerais sobre a navegação na disciplina. Já os alunos do EaD, tiveram uma participação muito efetiva, com 4.367 publicações, a maior parte com comentários a respeito do tema em questão. Ao final dos 4 fóruns, tivemos 11.272 publicações dos alunos do EaD e 1.469 dos alunos da modalidade presencial. É muito provável que essa diferença possa ser explicada pelo fato de os 
alunos oriundos de cursos EaD já estarem acostumados a participarem de fóruns de discussão como os sugeridos no curso.

$\mathrm{Na}$ tabela abaixo, apresentamos de maneira resumida a quantidade de participantes nos fóruns de discussão, com a divisão de participação dos estudantes por modalidade de ensino. Havendo, significativamente, uma maior participação dos estudantes que já realizam Cursos EaD.

Número total de participantes - fóruns de discussões

Participantes nos fóruns de discussões - Cursos presenciais 1.469

Participantes nos fóruns de discussões - Cursos EaD

\section{Conclusão}

A presente pesquisa teve como objetivo analisar dois aspectos fundamentais relacionados aos recursos educacionais presentes no curso Carreira S/A. A oferta e a análise da interação dos alunos com os recursos educacionais de um curso dessa magnitude representou considerável desafio em termos de gestão e de análise de dados, pois essa enorme quantidade de alunos gerou além de grande quantidade de dados, organização diferenciada para monitorar as questões operacionais do curso, como, por exemplo, responder as centenas de mensagens dos alunos sobre diversos assuntos e verificar os temas mais citados nos fóruns de discussão. Ainda que a figura do tutor não estivesse presente, os gestores do curso acompanhavam as necessidades "colocadas" nos fóruns, sem mediar as discussões. Foram a partir desses monitoramentos que algumas ações efetivaram-se, como por exemplo conceber os avisos criados durante o decorrer do curso que visavam a orientar os alunos nos aspectos relacionados às questões tecnicas. O desafio tecnológico também esteve presente, pois foi preciso considerar que alguns aspectos previstos pela equipe de produção do curso, como a integração de quizzes aos vídeos não funcionou como o esperado no LMS. Em relação, especificamente, aos quizzes, infelizmente uma incompatibilidade entre a versão da Kaltura e o Blackboard inviabilizou a atribuição das notas dos quizzes no boletim de cada participante. Os quizzes integrados teriam sido úteis, pois possibilitariam que as perguntas fossem colocadas, diaretamente, em qualquer ponto do vídeo, tornando este objeto de aprendizagem mais lúdico e assertivo.

Outro assunto que merece destaque diz respeito à participação dos alunos presenciais e EaD nos fóruns de discussão. Foi possível perceber que os alunos da modalidade EaD utilizaram o espaço com mais frequência e, efetivamente, para apresentaram dúvidas e colocações específicas sobre os assuntos sugeridos nas diversas linhas de discussões. 
Já os alunos da modalidade presencial, além de interagirem menos, utilizaram o espaço para esclarecer dúvidas a respeito de navegação e outros aspectos do material e do ambiente. Os alunos da modalidade EaD, nesse caso, foram mais participativos e tiveram menos dúvidas a respeito da utilização do Blackboard, provavelmente, por estarem mais habituados a utilizarem esse tipo de espaço para discussão e/ou ainda, por utilizarem com mais intensidade o LMS. Em termos de trabalhos futuros, sugerem-se novos estudos a respeito de possibilidades diferenciadas para a produção de vídeos para EaD, como aliar os já consagrados formatos e gêneros da televisão à produção pedagógica. Além disso, seria interessante que novas experimentações tecnológicas fossem efetivadas para integrar os quizzes presentes nas videoaulas aos mais diferentes AVAs e LMSs. Ainda que não faça parte do escopo do presente trabalho, os resultados obtidos nesta análise, apontam para a necessidade de análise do comportamente de alunos dos MOOCs, visto que em um universo de 181.677 matriculas, menos de $10 \%$, ou seja, apenas 12.741 alunos participaram, efetivamente, dos fóruns de discussão.

\section{Referências}

Allen, I., Seaman, J.E, \& Seaman, J. (2018). Online Learning Consortium., disponível em Online Report Card - Tracking Online Education in the United States, 2018: https://onlinelearningsurvey.com/reports/gradeincrease.pdf Acesso em 26 de julho de 2018.

BAKER, R. S. J.; ISOTANI, S.; DE CARVALHO, A. M. J. B. Mineração de dados educacionais: oportunidades para $\circ$ Brasil. Revista Brasileira de Informática na Educação, v.19, n. 2, p. 1-12, 2011.

Baturay, M. (2015). An overview of the world of MOOCs. Procedia - Social and Behavioral Sciences, 174, 427-433.

Guo, P., Kim, J., \& Rubin, R. (2014). How video production affects student engagement: An empirical study of mooc videos. Proceedings of the first ACM conference on Learning@ scale conference (pp. 41-50). Atlanta, Georgia, USA: ACM - Association for Computing Machiinery.

Hyman, P. (2012). In the Year of Disruptive Education. Communications of the acm, 55(12), 20-22.

INEP. (2016). Censo da educação superior 2016: notas estatísticas. Fonte: Instituto 
Nacional de Estudos e Pesquisas Educacionais Anísio Teixeira: http://download.inep.gov.br/educacao_superior/censo_superior/documentos/2016/notas sobre_o_censo_da_educacao_superior_2016.pdf. Acesso em 26 de julho de 2018.

Jordan, K. (2015). Massive Open Online Course Completion Rates Revisited: Assessment, Length and Attrition. The International Review of Research in Open and Distributed Learning, 16(3).

Kaltura. (2016). The State of Video in Education 2016: A Kaltura Report. Acesso em 20 de abril de 2016, disponível em Kaltura: https://corp.kaltura.com/sites/default/files/The\%2 0State\%20of\%20Video\%20in\%20Education\%202016\%20-\%20A\%20Kaltura\%20Report. pdf?alild=165316164

Khalil, M., \& Ebner, M. (2017). Clustering patterns of engagement in Massive Open Online Courses (MOOCs): the use of learning analytics to reveal student categories. Journal of Computing in Higher Education, 29(1), 1-19.

Khan, S. (2012). The one world schoolhouse: Education reimagined. New Yourk: Twelve.

Nanfito, M. (2014). MOOCs: Opportunities, impacts, and challenges: massive open online courses in colleges and universities. Createspace - Amazon.

PARDO, A.; KLOOS, C. D. Stepping out of the box: towards analytics outside the learning management system. In Proceedings of the 1st International Conference on Learning Analytics and Knowledge. Banff, Canada: ACM., p. 163-167, 2011.

Pomerol, J., Epelboin, Y., \& Thory, C. (2015). MOOCs: Design, Use and Business Models. John Wiley \& Sons. 